\title{
Smoking and common mental disorders in patients with chronic conditions: An analysis of data collected via a web-based screening system
}

Faith Matcham MSc ${ }^{1,2}$, Amy Carroll DClinPsy ${ }^{3}$, Natali Chung $\mathrm{MD}^{3}$, Victoria Crawford ${ }^{4}$, James Galloway $\mathrm{PhD}^{4,5}$, Anna Hames DClinPsy ${ }^{4}$, Karina Jackson $\mathrm{MSc}^{6}$, Clare Jacobson DClinPsy ${ }^{3}$, Dulka Manawadu MRCP ${ }^{4}$, Lance McCracken $\mathrm{PhD}^{7}$, John Moxham $\mathrm{MD}^{8}$, Lauren Rayner PhD ${ }^{1,2}$, Deborah Robson $\mathrm{PhD}^{9}$, Anna Simpson MSc ${ }^{1,2}$, Nicky Wilson $\mathrm{MSc}^{4}$, Matthew Hotopf $\mathrm{PhD}^{1,2}$.

1. Department of Psychological Medicine, Institute of Psychology, Psychiatry and Neuroscience, King's College London.

2. NIHR Biomedical Research Centre for Mental Health at the South London and Maudsley NHS Foundation Trust, King's College London.

3. Guy's and St. Thomas' NHS Foundation Trust, London

4. King's College Hospital NHS Foundation Trust, London

5. Department of Academic Rheumatology, King's College London

6. St. John's Institute of Dermatology, Guy's and St. Thomas' NHS Foundation Trust

7. Health Psychology Section, Psychology Department, Institute of Psychology, Psychiatry and Neuroscience, King's College London.

8. Department of Asthma, Allergy and Respiratory Science, King's College London.

9. National Addiction Centre, Addictions Department, King's College London.

Corresponding author: Faith Matcham, Weston Education Centre, 10 Cutcombe road, London SE5 9RJ. Faith.matcham@kcl.ac.uk.0207 8480868. 


\section{ABSTRACT}

Objective: Smoking is the largest preventable cause of death and disability in the UK and remains pervasive in people with mental disorders and in general hospital patients. We aimed to quantify the prevalence of mental disorders and smoking, examining associations between mental disorders and smoking in patients with chronic physical conditions.

Method: Data were collected via routine screening systems implemented across two London NHS Foundation Trusts. The prevalence of mental disorder, current smoking, nicotine dependence and wanting help with quitting smoking were quantified, and the relationships between mental disorder and smoking were examined, adjusting for age, gender and physical illness, with multiple regression models.

Results: A total of 7,878 patients were screened; $23.2 \%$ screened positive for probable major depressive disorder, and $18.5 \%$ for probable generalised anxiety disorder. Overall, $31.4 \%$ and $29.2 \%$ of patients with probable major depressive disorder or generalised anxiety disorder respectively were current smokers. Probable major depression and generalised anxiety disorder were associated with $93 \%$ and $44 \%$ increased odds of being a current smoker respectively. Patients with depressive disorder also reported higher levels of nicotine dependence, and the presence of common mental disorder was not associated with odds of wanting help with quitting smoking.

Conclusion: Common mental disorder in patients with chronic physical health conditions is a risk factor for markedly increased smoking prevalence and nicotine dependence. A general hospital encounter represents an opportunity to help patients who may benefit from such interventions.

\section{KEYWORDS}

Smoking, common mental disorders, chronic physical conditions, screening, comorbidity 


\subsection{INTRODUCTION}

Smoking is the largest preventable cause of disability and death in the UK, and one of the most costeffective methods of maintaining health and prolonging life is to encourage smoking cessation.[1] The prevalence of smoking continues to decline in the UK, having already dropped from $45 \%$ of all adults in 1974, to $19 \%$ of adults in 2013.[2] However this decline in smoking behaviour has not been mirrored amongst people with mental disorders: an estimated $37 \%$ of people with depression are current smokers, and people with a mental disorder smoke approximately $42 \%$ of all the cigarettes smoked by the English general population.[3] Similarly, data from a nationally representative US sample reports a slower decline in smoking rates between 2004-2011 in people with mental disorder in comparison to people without mental disorder. [4] The overlap between mental and physical illness is substantial, with $30 \%$ of the UK population having a long-term physical illness,[5] and $30 \%$ of those also have a comorbid mental disorder.[6] Smoking in general hospital patients is prevalent, with 1.1 million smokers treated per year in English hospitals, representing 2.6 million episodes of care.[7]

There are several effective methods of enhancing smoking cessation, including behavioural support and medication, which are offered free of charge within the National Health Service (NHS).[8] Evidence suggests that smoking cessation interventions can be effective are no less effective in smokers with common mental disorders. [9] A recent multicentre randomised controlled trial examining the impact of varenicline on smoking cessation rates in adults with stable current or past major depressive disorder has shown increased cessation with no adverse effects on symptoms of depression or anxiety. [9] Furthermore, staged intervention incorporating motivational feedback and psychological counselling can be effective for enhancing cessation in mental health outpatients. [10] Analysis of a randomised controlled trial examining the impact of counselling combined with Nicotine Replacement Therapy has also demonstrated no adverse mental health outcomes after quitting smoking in patients with clinical depression [11] and meta-analysis evidence suggests that cessation may reduce symptoms of depression and anxiety [12]. 
Despite the range of effective interventions available, smoking cessation rates in people with mental disorders are much lower than in the general population.[13-14] Depressed smokers are as willing to attempt cessation as non-depressed smokers, and severity of depression and history of depression recurrence are not associated with willingness to quit.[15-16] However smokers with mental disorders are less likely to be offered smoking cessation support and are frequently excluded from smoking cessation trials.[17] One hypothesis for this may be that healthcare professionals may perceive depressed smokers to be unwilling to quit, less able to quit, or have concerns that quitting may exacerbate symptoms of depression.[18] However an unsuccessful quit attempt has been previously associated with increased odds of experiencing lifetime depression and anxiety compared to non-quitters, and increased prevalence of current depression [19].

The frequency with which many patients with chronic physical conditions are required to attend hospital appointments means that a hospital encounter may be a potentially valuable intervention point. Experiencing a health scare, such as diagnosis of illness or acute health event, may provide a teachable moment to facilitate behaviour change, and result in smokers being more likely to engage in cessation interventions.[20] Smoking cessation in patients with chronic physical conditions is likely to be highly beneficial for improving health outcomes. However, as yet there has been limited assessment of smoking and mental health within the context of physical illness.

To the best of our knowledge, this will be the first paper to examine the relationships between mental and physical comorbidity and smoking, using screening data collected as part of routine clinical practice, representing a large heterogeneous secondary care sample. We aimed: 1) to describe the prevalence of mental disorders, current smoking status, and level of nicotine dependence in patients with chronic physical conditions; 2) to examine the associations between mental disorders and smoking prevalence and level of nicotine dependence in patients with chronic physical health conditions; and 3) to establish the association between mental health status and wanting help with quitting smoking in patients with chronic physical conditions. 


\subsection{METHOD}

\subsection{Procedure}

Data were collected via the Integrating Mental and Physical Healthcare: Research, Training and Services (IMPARTS) screening interface. [21] IMPARTS was designed to integrate the detection and management of mental disorders within physical healthcare environments; the web-based screening platform also facilitates the routine collection of patient-reported outcomes, and health behaviours, including smoking. IMPARTS provides this screening interface within a novel model of service delivery allowing physical health specialties to routinely monitor pertinent elements of mental and physical health, and health behaviours in real-time, and provide appropriate, tailored support and recommendations for onward referral. IMPARTS has been implemented within King's Health Partners (KHP), an Academic Health Science Centre in London, including a university (King's College London), two acute NHS Hospital Trusts (King's College Hospital (KCH) NHS Foundation Trust and Guy's and St. Thomas' (GSTT) NHS Foundation Trust), and an NHS Mental Health Trust (South London and the Maudsley NHS Foundation Trust).

Patients attending general hospital appointments were given an information sheet inviting them to complete a series of questionnaires whilst waiting for their appointment. The information sheet informed them that the information is being collected as part of their routine care at the hospital, and that the information they provide is confidential and will be dealt with anonymously if used for audit or research purposes. Informed consent is not required for these purposes. Patients completed a series of measures chosen by the clinical team and specific to their physical condition on an iPad. The data were then transferred immediately to their Electronic Patient Record (EPR), allowing the clinicians to review the results in real-time, facilitating discussion during the appointment. Positive screens for probable major depressive disorder (pMDD), suicidal ideation or probable generalised anxiety disorder (pGAD) were flagged, with appropriate care pathways and risk assessment procedures provided. The system has been designed to be fully embedded in clinical care, with existing staff taking responsibility for implementing screening, and managing the screening results, supported by ongoing training from the IMPARTS team when required. 
Twenty-one clinical services across 3 acute hospital sites were utilising the IMPARTS screening system at the time of these analyses. These represent services that have identified the need to standardise their collection of patient-reported outcomes, and approached the IMPARTS team requesting the implementation of routine screening in their clinics. These services range from large rheumatology and dermatology clinics, seeing hundreds of patients per week, to smaller services seeing less common physical conditions such as neuroendocrine tumours and endocarditis. The heterogeneity in services has resulted in vastly differing sample sizes for each chronic physical condition. For the purposes of this study, a pragmatic decision was made to include only services contributing data from over 100 unique patients between February 2011 (when the first service implemented IMPARTS) until June 2015. Patients were asked to complete questionnaires via the IMPARTS screening system at every hospital visit, however smoking data is only collected at one hospital visit per year. This analysis focused on the data collected at each patient's first screening encounter. Although primarily a method of collecting patient-reported outcomes for clinical care, IMPARTS has received ethical approval for anonymised data collected via the screening interface to be used for research purposes (REC: 12-SC-0422).

\subsection{Setting}

Data from 10 services were used in the current analysis. Pilot data collected from Rheumatology, Limb Reconstruction and Dermatology services show that uptake of screening was high, with the majority of patients attending appointments being successfully screened (62.5\%-98.2\%) and only a small proportion of patients declining screening (0.6\%-5.1\%).[21] The services contributing data to the current analysis were: adult congenital heart disease (ACHD); dermatology (a tertiary service comprising patients with hidradenitis suppurativa, psoriasis or eczema); dialysis (representing patients on haemodialysis in three satellite units); limb reconstruction; liver transition (adolescents or young adults with chronic liver disease or liver transplant); musculoskeletal physiotherapy; chronic pain (an interdisciplinary unit delivering intensive outpatient or residential treatments); rheumatology (representing the full range of rheumatic diseases); stroke (hyper-acute stroke outpatients); and teenage and young adult (TYA) cancer. 


\subsection{Measures}

The IMPARTS screening interface allows for a flexible and tailored battery of measures to be implemented in different services, depending on the physical condition. Given that the primary purpose of data collection via IMPARTS is for routine clinical care, the measures chosen are those of most clinical utility within the clinic setting. As such, they are necessarily brief, and the mental health questionnaires are limited to assessing common mental health problems (depression and anxiety). Although the questionnaire sets used in each service differ, depression, anxiety, and smoking are measured using the same outcome measures across all services, except for anxiety which was not measured in the pain service. Given the heterogeneity of the data collected for each physical condition, analysis was restricted to variables common to all physical conditions: age, gender, depression, anxiety, and smoking.

\subsubsection{Depression}

Depression was measured using the 9-item Patient Health Questionnaire (PHQ9; [22] which has been recommended by the National Institute for Health and Care Excellence (NICE) for use in adult patients with chronic physical health problems.[23] The PHQ9 has good sensitivity and specificity for identifying major depressive disorder in patients with physical health conditions,[24] and has good test-retest reliability. [25] To identify the presence of probable major depressive disorder (pMDD), the validated categorical algorithm was used. [24] pMDD was defined as scoring "more than half the days" or "nearly every day" within the last two weeks on at least one of the first two items of the PHQ-9 (low mood and a loss of interest in activities), and on at least five out of all nine symptoms. Item nine of the PHQ9 ("Thoughts that you would be better off dead or of hurting yourself in some way") was used to assess suicidal ideation, with patients identifying this symptom more than half the days in the past two weeks categorised as experiencing suicidal ideation contributing towards pMDD diagnosis if present at all. [24]

Patients scoring "not at all "or "several days" to all questions were categorised as having "No symptoms", and patients reporting symptoms "more than half the days" or "nearly every day" but not reaching criteria for pMDD were coded as having "some symptoms". This resulted in a final 
depression independent variable coded with the following categories: 0 "No symptoms"; 1 "Some symptoms"; 2 "pMDD".

\subsubsection{Anxiety}

Anxiety was assessed using the 7-item Generalised Anxiety Disorder (GAD7) questionnaire. [26] This measure has good reliability, sensitivity and specificity, as well as criterion, construct, factorial and procedural validity. [26] A score of 10 or more on the GAD7 identified cases of probable Generalised Anxiety Disorder (pGAD); scores ranging 5-9 indicated the presence of some anxiety symptoms. This results in a final anxiety independent variable coded with the following categories: 0 "no symptoms" (scores <5); 1 "some symptoms" (scores 5-9); and 2 "pGAD" (scores $\geq 10$ ).

\subsubsection{Smoking}

Prevalence of current smoking was assessed by asking patients if they currently smoke (providing yes or no answers). The severity of nicotine dependence for self-reported smokers was assessed using the Heaviness of Smoking Index (HSI).[27] This two item measure asks how many cigarettes, cigars or rollups they smoke per day (cigarettes per day; CPD); and how soon after waking up they smoke their first cigarette, allowing one of the following categories to be selected: within 5 minutes; 6-30 minutes; 31-60 minutes; after 60 minutes (time to first cigarette; TTFC). CPD and TTFC can be combined to form the HSI through scoring CPD from 0 (10 or less) to 3 (31 or more), scoring TTFC from 0 (after 60 minutes) to 3 (within 5 minutes), and summing the total. This results in a score from 0-6, with higher scores indicating heavier smoking levels. Since March 2014 current smokers were also asked if they wanted help with quitting smoking using a one-item question ("Would you like help with quitting smoking") with "Yes/No" answer options. This question was added in later to coincide with the hospitals introducing new smoking cessation services, with the aim of identifying patients who would be willing to receive onward referral to smoking cessation services.

\subsection{Statistical Analysis}

Percentages, means and standard deviations were used to describe sample demographics and prevalence of psychological morbidity and smoking and level of nicotine dependence. The IMPARTS 
system does not allow patients to complete a questionnaire leaving any answers omitted, therefore there were no missing data. All analyses were run using Stata v.14.

Aim 1: Describing the prevalence of mental disorder, current smoking status and level of nicotine dependence in patients with chronic physical health conditions.

Prevalence of mental disorders and smoking were expressed as percentages, with $95 \%$ confidence intervals $(95 \% \mathrm{Cl})$. Descriptions of the smoking characteristics of patients with and without pMDD or pGAD were compared using Chi-square tests for categorical data (current smoking status, wanting help with quitting) and analysis of variance (ANOVA) for continuous data (nicotine dependence).

Aim 2: The association between mental disorder and smoking prevalence and level of nicotine dependence in patients with chronic physical health conditions.

A multiple logistic regression model assessed the relationship between the independent variables (depression and anxiety) and dependent variable (current smoking status). Suicidal ideation, gender, age and physical condition were also included as covariates. As anxiety was not measured in the pain service, the model was created in two stages: 1) all independent variables and covariates were entered into the model simultaneously to report the associations between depression, age, gender and suicidal ideation and odds of being a current smoker; 2) the model was re-run excluding physical condition as a covariate to report the association between anxiety and odds of being a current smoker, adjusting for age, gender, depression and suicidal ideation. Tests of linear trend were included to provide a p-value for a linear association between increased depression and anxiety severity and odds of being a current smoker.

The same variable entry method and adjustment for covariates was used in a multivariate linear regression model examining the linear dependent variable: nicotine dependence. Tests of linear trend were included to provide a p-value for a linear association between increased depression and anxiety severity and level of nicotine dependence. 
Aim 3: the association between mental health status and wanting help with quitting smoking in patients with chronic physical conditions.

A multiple logistic regression model assessed the relationship between the independent variables (depression and anxiety) and dependent variable (wanting help with quitting). Suicidal ideation, gender, age and physical condition were also included as covariates. The same variable entry method and adjustment for covariates were used, as described for aim 2. Tests of linear trend were included to provide a p-value for linear association between increased depression and anxiety severity and odds of wanting help with quitting smoking.

\subsection{RESULTS}

\subsection{Sample Statistics}

The study sample is described in Table 1. In total, data from 7878 outpatients were included, representing 10 chronic physical conditions. The overall mean age was 44.8 years (SD=16.2), and $57.8 \%$ of the overall sample were female. Mean ages and proportion of female patients varied across the physical conditions, in accordance with expected varying patient demographics: the mean ages in services seeing exclusively young adults (liver transition and TYA cancer) were 18.1 and 21.7 years respectively; conditions typically affecting working age adults had mean ages of 43.9, 44.8, 43.8, 45.8, and 50.0 years (dermatology, limb reconstruction, MSK physiotherapy, pain and rheumatology respectively).

\subsection{The prevalence of common mental disorders and smoking}

The combined prevalence of pMDD was $23.2 \%$ (95\%Cl: $22.2 \%-24.1 \%)$, with $6.1 \%(95 \% \mathrm{Cl}: 5.6 \%$ $6.6 \%$ ) of patients experiencing suicidal ideation and the prevalence of pGAD across the physical conditions was $18.5 \%$ (95\%Cl: $17.6 \%-19.4 \%)$ (Table 1). The prevalence of current smoking overall was $19.6 \%$ (95\%Cl: $18.7 \%-20.5 \%)$ and the overall mean $\mathrm{HSI}$ was $1.7(\mathrm{SD}=1.5)$. Overall, $37.8 \%$ (95\%Cl: $34.9 \%-40.9 \%)$ of current smokers wanted help with quitting smoking.

A significantly larger percentage of patients with pMDD were current smokers; $31.4 \%$, in comparison to only $20 \%$ of patients with some symptoms, and $15.2 \%$ of patients with no depression symptoms 
$\left(\mathrm{Chi}^{2}=223.1\right.$, d.f. $\left.=2, \mathrm{p}<0.001\right)$. Smokers with $\mathrm{pMDD}$ also reported a HSI score of $2.4(\mathrm{SD}=1.2)$, in comparison to $2.2(\mathrm{SD}=1.1)$ and $2.1(\mathrm{SD}=1.2)$ in patients with some and no symptoms respectively $(F(2,1202)=8.1, p<0.001)$. Also, $43.4 \%$ of smokers with pMDD wanted help with quitting, in comparison to only $37.9 \%$ of smokers with some depressive symptoms and $34.2 \%$ of smokers with no depression $\left(\mathrm{Chi}^{2}=7.5\right.$, d.f. $\left.=2, \mathrm{p}=0.02\right)$. Similarly, a significantly larger percentage of patients with pGAD were current smokers; $29.2 \%$, in comparison to $15 \%$ of patients with some anxiety symptoms, and $15.6 \%$ of patients with no anxiety symptoms $\left(\mathrm{Chi}^{2}=128.2\right.$, d.f. $\left.=2, \mathrm{p}<0.001\right)$. Smokers with pGAD also reported a HSI score of $2.3(\mathrm{SD}=1.2)$, in comparison to $2.2(\mathrm{SD}=1.1)$ and $2.1(S D=1.1)$ in patients with some and no anxiety symptoms respectively $(F(2,960)=1.7, p<0.01)$. Also, $43.1 \%$ of smokers with pGAD wanted help with quitting smoking, in comparison to only $40.5 \%$ of smokers with some symptoms and $33.5 \%$ of smokers with no anxiety $\left(\right.$ Chi $^{2}=7.06$, d.f. $=2$, $\mathrm{p}=0.03)$.

$\gg>$ TABLE $1<<<$

\subsection{The associations between common mental disorders and smoking prevalence}

Table 2 shows the results of a multiple logistic regression analysis examining the associations between depression, anxiety and suicidal ideation and current smoking status, adjusting for age, gender and physical condition.

In comparison to patients with no symptoms of depression, patients with some symptoms had $46 \%$ greater odds of being a current smoker and patients with pMDD had 93\% higher odds of being a current smoker. This was statistically significant in a test of linear trend $(\mathrm{OR}=1.93, \mathrm{SE}=0.21$ $\mathrm{p}<0.001$; Table 2). Patients with $\mathrm{pGAD}$ had $44 \%$ increased odds of being a current smoker, in comparison to patients with no anxiety symptoms, in a test of linear trend ( $p=0.001$; Table 2$)$.

$>>$ TABLE $2<<<$

\subsection{The association between common mental disorders and nicotine dependence}

Table 3 shows the results of a multiple linear regression analysis examining the associations between depression, anxiety and suicidal ideation and nicotine dependence, adjusting for age, 
gender and physical condition. Patients with some symptoms of depression and pMDD had higher HSI scores, which was statistically significant in a test of linear trend $(p<0.001)$. There were no significant differences in nicotine dependence between patients with and without suicidal ideation or between anxiety categories.

$>>$ TABLE $3<<<$

\subsection{The association between mental health and wanting help to quit smoking}

Measurement of wanting help with quitting smoking was added in March 2014, making this data available only for the 1015 most recently screened smokers. Table 4 shows the results of a multivariate logistic regression model assessing the association between depression, anxiety and suicidal ideation and odds of wanting help with quitting smoking, adjusting for age, gender, depression category, suicidal ideation and anxiety category, adjusted for chronic physical condition.

Neither depression nor anxiety were associated with odds of wanting help with quitting smoking. $>>$ TABLE $4<<<$

\subsection{DISCUSSION}

This paper highlights the potential of embedding routine patient reported outcomes into clinical practice, promoting research as an outgrowth of patient care. Using data collected as part of routine care fosters research and audit with minimal intrusiveness for patients. We have identified that comorbid depression and anxiety are associated with increased smoking prevalence in patients with chronic physical health conditions: $31.4 \%$ of patients with comorbid depression and $29.2 \%$ of patients with comorbid anxiety are self-reported current smokers. These estimates are substantially higher than the $19 \%$ smoking prevalence reported in the UK general population. [2] Patients with comorbid mental disorder also demonstrated higher levels of nicotine dependence, and were no less likely to want help with quitting smoking than patients without psychological comorbidity. Indeed, patients with suicidal ideation reported increased odds of wanting help with quitting smoking. 
Despite reporting a higher level of smoking prevalence than the general population, [2] we have identified a lower prevalence estimate than has been previously reported in a general hospital sample. [7] This could be for several reasons: it may reflect our focus on long-term physical conditions managed in secondary care. An unselected hospital sample would be expected to have a larger proportion of chronic lung diseases and ischaemic heart disease patients, in whom smoking prevalence would be predictably higher [28]. Additionally, self-report smoking status may underestimate actual smoking, [29] particularly in smokers at risk of cardiovascular disease. [30] This under-reporting of smoking may be exacerbated in the current context. Patients are attending a hospital for a physical health condition; they may have a long-standing relationship with their healthcare professional (HCP) and they know their HCP will be viewing their screening results. [21] Patients who smoke may have already been encouraged to quit by their $\mathrm{HCP}$, or may not want to reveal that they are still smoking.

Services seeing exclusively younger patients, or patients usually diagnosed during childhood show the lowest smoking prevalence (liver transition 12.4\%, ACHD 10.6\% and TYA oncology $12.8 \%$ ). This finding is at odds with the wider literature. There is evidence highlighting increased engagement with health risk behaviours, such as smoking, antisocial behaviour and cannabis use in adolescents with physical conditions or disability. [31] Future research specifically examining this question may be beneficial, to improve understanding of the complex relationship between health and behaviour in childhood and adolescence.

The notably high proportion of patients reporting wanting help with quitting smoking $(43.4 \%$ of depressed patients and $43.1 \%$ of anxious patients) indicates that patients may find a hospital encounter useful for obtaining support with quitting smoking. This highlights the need for integrated smoking cessation support within general hospital environments. Early identification of smoking status and nicotine dependence is quick, simple, and is crucial for timely and appropriate intervention. A hospital encounter may act as an essential point of contact for smokers with mental and physical comorbidity, who may benefit the most from prompt smoking cessation support. Metaanalysis evidence has found a significant association between smoking cessation and reduced 
symptoms of depression and anxiety.[12] Both smoking and common mental disorder are related to worsened long-term physical health outcomes, [32] reduced quality of life [33] and increased mortality [34] in chronic diseases, therefore encouraging smoking cessation as part of routine clinical care serves a dual purpose in improving physical and mental health outcomes, and reducing risk of premature mortality. [35-37]

The collection of patient reported outcomes via tablet devices lends itself to the delivery of einterventions. Recently, patients reporting wanting help with quitting smoking via IMPARTS have been asked if they would like to sign-up to a text-based smoking cessation intervention: Txt2Stop.[38] If they consent, they are automatically sent a text message with details on how to sign up. The extent to which comorbidity and embedded screening can predict uptake of, adherence to, and outcomes from this form of intervention need to be assessed. Use of screening systems such as IMPARTS could revolutionise patient recruitment into trials, using routinely collected patient reported outcomes to identify eligible and motivated patients, and allow easy measurement of "consent for contact" for research purposes. [39]

\subsection{Limitations}

The data presented here comes from outpatients attending appointments for chronic physical health conditions across a range of specialist services. Whilst it is possible that the patients with highest levels of physical and mental health needs may not be captured using the in-clinic screening system, our results are likely to be more representative of the general outpatient population than research relying on traditional research consent procedures, which typically under-represent ethnic minorities and individuals from low socioeconomic status, [40] leading to questionable generalisability of findings. [41] Pilot data collected in several of these services (rheumatology, limb reconstruction and dermatology) suggest that the proportion of patients screened can vary across services (98.2\%$62.5 \%$ ), depending on the structure of the clinics and resources available.[21] We therefore have no information about the mental health and smoking status of the patients who were not screened and cannot assess any systematic differences between screened vs. non-screened patients. Moreover, 
we do not have information on patients who only partially completed screening, and reasons for noncompletion, such as a loss of internet connectivity.

The self-report of socially undesirable behaviours such as tobacco smoking may be subject to reporting bias, particularly in groups of people that are receiving care for smoking-related diseases. Estimates based on self-report are usually lower when compared with those established by biochemical measures such as salivary or urinary cotinine, however the extent of the difference in prevalence estimates of smoking status assessed via self-report or biochemical measures vary by study sample and country, with estimates ranging between $0.3 \%$ and $4.4 \%$ [42-43]. Biochemical measures are generally used in research populations rather than routine clinical practice, where resource constraints may preclude routine use of biochemical measures.

The Heaviness of Smoking Index used to assess severity of nicotine dependence is well validated in daily smokers, however the validity of this measure has not yet been reported for non-daily smokers [44]. The IMPARTS smoking measure does not capture daily vs non-daily smokers and therefore the validity of the HSI to indicate nicotine dependence in our sample is unclear.

Mining health records for research purposes is becoming more common, particularly with increasing pressure on research and clinical budgets.[45] However this methodology brings with it limitations in the quality of data available. Over $50 \%$ of the patients in our dataset had missing ethnicity data, due to data omissions from clinical records, spelling and typing errors, as well as inconsistencies in coding schemes between hospital sites. Whilst it would be preferable to be able to include sociodemographic information such as ethnicity and social deprivation, these data were not consistently available and reliably up-to-date. Similarly, the heterogeneous patient groups used in this analysis precluded inclusion of disease-related variables such as disease severity or comorbidity, which may also have been useful additions to our analysis.

\subsection{Conclusion}

This research has used data collected as part of routine clinical practice to examine the relationships between mental and physical comorbidity and smoking. Experiencing depression or anxiety is a risk 
factor for increased smoking prevalence and nicotine dependence in patients with chronic physical health conditions, but are not associated with reduced odds of wanting help with quitting smoking. These results have several implications. Common mental disorders are associated with substantially worsened longitudinal disease outcomes [32], to which smoking contributes. Patients with common mental disorders can have excellent outcomes from smoking cessation therapies, and a general hospital encounter represents an opportunity to access patients who may benefit from such interventions.

\section{STATEMENT OF CONTRIBUTION}

All authors have sufficiently contributed to and approved the final version of the manuscript and it has not been published elsewhere.

\section{ACKNOWLEDGEMENTS}

We acknowledge all members of clinical staff for their efforts facilitating IMPARTS screening and the IMPARTS research oversight committee for reviewing and approving this research project.

\section{FUNDING}

This study was supported by King's Health Partners and the National Institute for Health Research (NIHR) Biomedical Research Centre for Mental Health at the South London and Maudsley NHS Foundation Trust and Institute of Psychiatry, King's College London.

\section{DECLARATION OF INERESTS}

None 


\section{REFERENCES}

1 Royal College of Physicians. Forty fatal years: A review of the 40 years since the publication of the 1962 Report of the Royal College of Physicians on Smoking and Health. London, UK: 2002. http://www.ash.org.uk/files/documents/ASH 206.pdf (Accessed October 2015).

2 Action on Smoking and Health. Smoking statistics: Who smokes and how much. July 2015. http://ash.org.uk/files/documents/ASH 106.pdf (Accessed October 2015).

3 National Centre for Social Research. Cigarette smoking and mental health in England: Data from the Adult Psychiatric Morbidity Survey 2007. https://natcen.ac.uk/media/21994/smoking-mental-health.pdf. (Accessed May 2015).

4 Cook B, Wayne G, Kafali E, et al. Trends in smoking among adults with mental illness and association between mental health treatment and smoking cessation. JAMA. 2014;311:172-82. Doi:10.1001/jama.2013.284985.

5 Department of Health. Ten things you need to know about long term conditions. April 2008.

http://webarchive.nationalarchives.gov.uk/+/www.dh.gov.uk/en/Healthcare/Longtermco nditions/DH 084294 (Accessed June 2015).

6 Cimpean D, Drake RE. Treating co-morbid chronic medical conditions and anxiety/depression. Epidemiol Psychiatr Sci. 2011;20(2):141-50. Doi: 10/1017/S2045796011000345.

7 Szatkowski L, Murray R, Hubbard R, et al. Prevalence of smoking among patients treated in NHS hospitals in England in 2010/2011: A national audit. Thorax. 2015;70:498-500. Doi: 10.1136/thoraxjnl-2014-206285.

8 Bauld L, Bell L, McCullough L, et al. The effectiveness of NHS smoking cessation services: a systematic review. J Public Health (Oxf) 2010;32:71-82.Doi: 10.1093/pubmed/fdp074.

9 Anthenelli RM, Morris C, Ramey TS, et al. Effects of varenicline on smoking cessation in adults with stably treated current or past major depression: A randomized trial. Ann Intern Med 2013;159(1):390-400. Doi: 10.1093/pubmed/fdp074.

10 Hall SM, Tsoh JY, Prochaska JJ, et al. Treatment for cigarette smoking among depressed mental health outpatients: A randomized clinical trial. Am $J$ Public Health 2006;96(10):1808-14. Doi: 10.2105/AJPH.2005.080382.

11 Prochaska JJ, Hall, Tsoh JY, et al. Treating tobacco dependence in clinically depressed smokers: effect of smoking cessation on mental health functioning. Am J Public Health 2008;98 (3):446-8. Doi: 10.2105/AJPH.2006.101147.

12 Taylor G, McNeill A, Girling A, et al. Change in mental health after smoking cessation: systematic review and meta-analysis. BMJ 2014;348:g1151.Doi: http://dx.doi.org/10.1136/bmi.g1151.

13 Glasheen C, Hedden SL, Forman-Hoffman VL, et al. Cigarette smoking behaviors among adults with serious mental illness in a nationally representative sample. Ann Epidemiol 2014;24(10):776-80. Doi: 10.1016/j.annepidem.2014.07.009. 
14 Lasser K, Boyd J, Woolhandler S, et al. Smoking and mental illness: A population-based prevalence study. JAMA 2000;284(20):2606-2610. Doi: 10.1001/jama.284.20.2606.

15 Prochaska JJ, Rossi JS, Redding CA, et al. Depressed smokers and stage of change: implications for treatment interventions. Drug Alcohol Depend 2004;76(2):143-51. Doi: 10.1016/i.drugalcdep.2004.04.017.

16 Lawrence D, Mitrou F, Zubrick SR. Non-specific psychological distress, smoking status and smoking cessation: United States National Health Interview Survey 2005. BMC Public Health 2011;11:256.doi: 10.1186/1471-2458-11-256.

17 Jack LM, Swan GE, Thompson E, et al. Bupropion SR and smoking cessation in actual practice: methods for recruitment, screening, and exclusion for a field trial in a managedcare setting. Prev Med (Baltim) 2003;36(5):585-93. Doi: 10.1016/S0091-7435(03)00011$\underline{2}$.

18 Lembke A, Johnson K, DeBattisa C. Depression and smoking cessation: does the evidence support psychiatric practice? Neuropsychiatr Dis Treat 2007;3(4):487-93.

19 Boudreaux E, Baumann B, Camargo C, et al. Changes in smoking associated with an acute health event: Theoretical and practical implications. Ann Behav Med 2007;33(2):189-99.

20 Demark-Wahnefried W, Aziz N, Rowland J, et al. Riding the crest of the teachable moment: Promoting long-term health after the diagnosis of cancer. $J$ Clin Oncol 2005;23(24):5814-30. Doi: 10.1200/JCO.2005.01.230.

21 Rayner L, Matcham F, Hutton J, et al. Embedding integrated mental health assessment and management in general hospital settings: feasibility, acceptability and the prevalence of common mental disorder. Gen Hosp Psychiatry 2014;36(3):318-24. Doi: 10.1016/i.genhosppsych.2013.12.004.

22 Spitzer RL, Williams JBW, Kroenke K, et al. Utility of a New Procedure for Diagnosing Mental Disorders in Primary Care. JAMA 1994;272(22):1749-56. Doi: 10.1001/jama.1994.03520220043029.

23 National Institute for Health and Care Excellence. Depression in adults with a chronic physical health problem: Treatment and management. CG91. https://www.nice.org.uk/guidance/cg91.

24 Löwe B, Spitzer RL, Gräfe K, et al. Comparative validity of three screening questionnaires for DSM-IV depressive disorders and physicians' diagnoses. J Affec Disord 2004;78(2):131-40. Doi: 10.1016/S0165-0327(02)00237-9.

25 Bakker IM, Terluin B, van Marwijk HWJ, et al. Test-retest reliability of the PRIME-MD: limitations in diagnosing mental disorders in primary care. Eur $J$ Public Health 2009;19:303-7. Doi: 10.1093/eurpub/ckn149

26 Spitzer RL, Kroenke K, Williams JBW, et al. A brief measure for assessing generalized anxiety disorder: the GAD-7. Arch Intern Med 2006;166(10):1092-7. Doi: 10.1001/archinte.166.10.1092.

27 Heatherton TF, Kozlowski LT, Frecker RC, et al. Measuring the Heaviness of Smoking: using self-reported time to the first cigarette of the day and number of cigarettes smoked per day. Addiction 1989;84(7):791-800. Doi: 10.1111/j.1360-0443.1989.tb03059.x. 
28 Mannino DM, Buist AS. Global burden of COPD: Risk factors, prevalence and future trends. Lancet 2007;370(9589):765-73. Doi: 10.1016/S0140-6736(07)61380-4.

29 Connor Gorbor S, Schofield-Hurwitz S, Hardt J. The accuracy of self-reported smoking: a systematic review of the relationship between self-reported and cotinine-assessed smoking status. Nicotine Tob Res 2009;11(1):12-24. Doi: 10.1093/ntr/ntn010.

30 Pell JP, Cobbe SM, Haw SJ, et al. Validity of self-reported smoking status: Comparison on patients admitted to hospital with acute coronary syndrome and the general population. Nicotine Tob Res 2008;10(5):861-6. Doi: 10.1080/14622200202023858.

31 Suris JC, Michaud PA, Akre C, Sawyer SM. Health risk behaviors in adolescents with chronic conditions. Pediatrics 2008;122(5):1113-8. Doi: 10.1542/peds.2008-1479.

32 Matcham F, Norton S, Scott DL, et al. Symptoms of depression and anxiety predict treatment response and long-term physical health outcomes in rheumatoid arthritis: Secondary analysis of a randomized controlled trial. Rheumatology (Oxford) 2016;55(2):268-78. doi:10.1093/rheumatology/kev306

33 Alonso J, Angermeyer C, Bernet S, et al. Disability and quality of life impact of mental disorders in Europe: results from the European Study of the Epidemiology of Mental Disorders (ESEMeD) project. Acta Psychiatr. Scand. Suppl 2004;109(204):38-46. Doi: 10.1111/j.1600-0047.2004.00329.x

34 Ang DC, Choi H, Kroenke K. Comorbid depression is an independent risk factor for mortality in patients with rheumatoid arthritis. J Rheumatol 2005;32(6):1013-9.

35 Critchley JA, Capewell S. Mortality risk reduction associated with smoking cessation in patients with coronary heart disease: a systematic review. JAMA 2003;290(1): 86-97. Doi: 10.1001/jama.290.1.86

36 Godtfredsen NS. Smoking reduction, smoking cessation, and mortality: A 16-year followup of 19,732 men and women from the Copenhagen Centre for Prospective Population Studies. Am. J. Epidemiol 2002;156(11): 994-1001.

37 Mohiuddin SM. Intensive smoking cessation intervention reduces mortality in high-risk smokers with cardiovascular disease. Chest 2007;131(2):446.

38 Free C, Whittaker R, Knight R, et al. Txt2stop: a pilot randomised controlled trial of mobile phone-based smoking cessation support. Tob Control 2009;18(2):88-91.

39 Callard F, Broadbent M, Denis M, et al. Developing a new model for patient recruitment in mental health services: a cohort study using Electronic Health Records. BMJ Open 2014;4(12):p.e005654.

40 Ford JG, Howerton MW, Lai GY, et al. Barriers to recruiting underrepresented populations to cancer clinical trials: As systematic review. Cancer 2008;112(2):228-42.

41 Rothwell PM. External validity of randomised controlled trials: 'to whom do the results of this trial apply? Lancet 2005;368(9453):82-93.

42 Wong SL, Shields M, Meatherdale S, et al. Assessment of validity of self-reported smoking status. Health Reports 2012;23(1):47-53.

43 West R, Zatonski W, Przewozniak K, et al. Can we trust national smoking prevalence figures? Discrepancies between biochemically assessed and self-reported smoking rates in three countries. Cancer Epidemiol Biomarkers Prev 2007;16(4):820-2. 
44 Etter J-F, Duc TV, Perneger TV. Validity of the Fagerstrom test for nicotine dependence and of the Heaviness of Smoking Index among relatively light smokers. Addiction 1999;94(2):269-281.

45 Jensen PB, Jensen LJ, Brunak S. Mining electronic health records: towards better research applications and clinical care. Nat. Rev. Genet 2012;13(6):395-405. 
Table 1. Description of study sample, and prevalence of pMDD, suicidal ideation, pGAD and smoking.

\begin{tabular}{|c|c|c|c|c|c|c|c|c|c|c|c|c|}
\hline & & Combined & ACHD & Dermatology & Dialysis & LR & LT & MSK & Pain & Rheumatolog & Stroke & TYA Cancer \\
\hline \multicolumn{2}{|l|}{ Total N } & 7878 & 667 & 1613 & 228 & 513 & 178 & 789 & 1093 & 2475 & 166 & 156 \\
\hline \multicolumn{2}{|l|}{ Age, $M(S D)$} & $44.8(16.2)$ & $31.6(13.5)$ & $43.9(14.6)$ & $55.5(16.5)$ & $44.8(14.9)$ & $18.1(1.7)$ & $43.8(14.0)$ & ) $45.8(12.2)$ & $50.0(15.6)$ & $62.7(15.1)$ & $21.7(2.5)$ \\
\hline \multirow{3}{*}{ Age Category, $\mathrm{N}(\%)$} & $33-45$ years & $2026(25.7)$ & $131(19.6)$ & $496(30.8)$ & $30(13.2)$ & $145(28.3)$ & $0(0.00)$ & 247 (31.3) & $348(31.8)$ & $616(24.9)$ & $13(7.8)$ & $0(0.0)$ \\
\hline & $46-55$ years & $1786(22.7)$ & $55(8.3)$ & $375(23.3)$ & $65(28.5)$ & $123(24.0)$ & $0(0.00)$ & $183(23.2)$ & $357(32.7)$ & $595(24.0)$ & $33(19.9)$ & $0(0.0)$ \\
\hline & $>55$ years & $2034(25.8)$ & $51(7.7)$ & $349(21.6)$ & $110(48.3)$ & $123(24.0)$ & $0(0.00)$ & $164(20.8)$ & $222(20.3)$ & $902(36.4)$ & $113(68.1)$ & $0(0.0)$ \\
\hline \multicolumn{2}{|l|}{ Female Gender, N(\%) } & $4553(57.8)$ & $336(50.4)$ & $720(44.6)$ & $98(43.0)$ & $134(26.1)$ & $94(52.8)$ & $480(60.9)$ & $736(67.3)$ & $1806(73.0)$ & $78(47.0)$ & $71(45.5)$ \\
\hline \multirow[t]{3}{*}{ Depression, $\mathrm{N}(\%)^{\wedge}$} & No Symptoms & $4951(63.2)$ & $577(86.5)$ & 1166 & $122(53.5)$ & $341(66.6)$ & $132(66.6)$ & $500(65.8)$ & 305 (27.9) & $1587(64.3)$ & $127(76.5)$ & $94(60.7)$ \\
\hline & Some Symptoms & s 1070 (13.7) & $62(9.3)$ & $176(10.9)$ & $85(38.3)$ & $73(14.3)$ & $25(14.3)$ & $129(17.0)$ & $130(11.9)$ & $325(13.2)$ & $22(13.3)$ & $43(27.7)$ \\
\hline & pMDD & 1815 (23.2) & $28(4.2)$ & $271(16.8)$ & $21(9.2)$ & $98(19.1)$ & $18(10.3)$ & $131(17.2)$ & $658(60.2)$ & $555(22.5)$ & $17(10.2)$ & $18(11.6)$ \\
\hline \multicolumn{2}{|c|}{ Suicidal Ideation, $\mathrm{N}(\%)$} & $471(6.1)$ & $7(1.1)$ & $66(4.1)$ & $8(5.2)$ & $27(5.3)$ & $4(2.3)$ & $35(4.6)$ & $160(14.6)$ & $150(6.1)$ & $9(5.4)$ & $5(3.2)$ \\
\hline \multirow[t]{2}{*}{ Anxiety, $\mathrm{N}(\%)^{\wedge}$} & No Symptoms & $5141(75.9)$ & $583(87.4)$ & $1252(77.6)$ & $168(73.7)$ & $382(74.6)$ & $135(77.1)$ & $596(75.8)$ & - & $1795(76.7)$ & $130(78.3)$ & $100(64.5)$ \\
\hline & pGAD & $1250(18.5)$ & $55(8.3)$ & $295(18.3)$ & $24(10.5)$ & $104(20.3)$ & $26(14.9)$ & $154(19.6)$ & - & $553(22.4)$ & $21(12.7)$ & $18(11.6)$ \\
\hline \multicolumn{2}{|l|}{ pMDD and pGAD } & $825(10.5)$ & $21(3.2)$ & $200(12.4)$ & $12(5.3)$ & $69(13.5)$ & $11(6.2)$ & $102(12.9)$ & - & $386(15.6)$ & $10(6.0)$ & $14(9.0)$ \\
\hline \multicolumn{2}{|l|}{ Current Smoker, N(\%) } & $1543(19.6)$ & $71(10.6)$ & $404(25.1)$ & $23(10.1)$ & $108(21.1)$ & $22(12.4)$ & $141(17.9)$ & $314(28.7)$ & $416(16.8)$ & $24(14.5)$ & $20(12.8)$ \\
\hline \multicolumn{2}{|l|}{$\mathrm{HSI}, \mathrm{M}(\mathrm{SD})^{\wedge \wedge}$} & $1.7(1.5)$ & $1.0(1.3)$ & $1.7(1.4)$ & $1.6(1.4)$ & $1.6(1.6)$ & $0.9(1.3)$ & $1.5(1.4)$ & $2.1(1.5)$ & $1.5(1.4)$ & $1.8(1.6)$ & $1.0(1.3)$ \\
\hline \multicolumn{2}{|c|}{ Wants help with quitting, $N(\%)^{\wedge \wedge}$} & $384(37.8)$ & $18(52.9)$ & 99 (34.4 & $6(26.1)$ & $25(48.1)$ & $1(5.3)$ & $44(31.2)$ & 78 (44.3) & $98(40.0)$ & $12(50.0)$ & $3(23.1)$ \\
\hline
\end{tabular}

ACHD Adult Congenital Heart Disease. LR Limb Reconstruction. LT Liver Transition. MSK Musculoskeletal Physiotherapy. TYA Teenage and Young Adult. MDD Major depressive disorder. GAD Generalised anxiety disorder. HSI Heaviness of Smoking Index. ^ ${ }^{\wedge}$ Depression measured in 6505 patients, anxiety measured in $5437 .{ }^{\wedge} \wedge$ Asked in $1427(92.4 \%)$ of current smokers. 
Table 2. Logistic regression model showing the relationship between depression, anxiety and suicidal ideation and current smoking status, adjusting for and age, gender, and physical condition.

\begin{tabular}{llllll}
\hline Variable & & OR & SE & p & $95 \%$ Cl \\
\hline Depression & No symptoms & - & - & - & - \\
& Some symptoms & 1.46 & 0.14 & & $1.21,1.77$ \\
& pMDD & 1.93 & 0.21 & & $1.56,2.39$ \\
Anxiety^ & & & & $0.001^{*}$ & \\
& No symptoms & - & - & - & - \\
& Some symptoms & 0.79 & 0.12 & & $0.58,1.07$ \\
Suicidal Ideation & pGAD & 1.44 & 0.15 & & $1.18,1.76$ \\
& No & - & - & - & - \\
Age & Yes & 1.24 & 0.18 & 0.13 & $0.94,1.64$ \\
Gender & & 0.98 & 0.00 & $<0.001$ & $0.98,0.99$ \\
& Male & - & - & - & - \\
& Female & 0.75 & 0.05 & $<0.001$ & $0.66,0.86$
\end{tabular}

${ }^{*} p$ value for trend. ^Not adjusted for physical condition. pMDD probable Major Depressive Disorder. pGAD probable Generalised Anxiety Disorder

Table 3. Linear regression model showing the relationship between depression, anxiety, suicidal ideation and nicotine dependence, adjusting for age, gender and physical condition (in current smokers, $\mathrm{N}=1318$ ).

\begin{tabular}{llllll}
\hline Variable & & $\mathbf{b}$ & $\mathbf{S E}$ & $\mathbf{p}$ & $\mathbf{9 5 \%} \mathbf{C l}$ \\
\hline Depression & No symptoms & - & - & $<0.001^{*}$ & - \\
& Some symptoms & 0.24 & 0.13 & & $-0.01,0.48$ \\
& pMDD & 0.49 & 0.13 & & $0.23,0.75$ \\
Anxiety^ & No symptoms & - & - & $0.91^{*}$ & - \\
& Some symptoms & 0.02 & 0.21 & & $-0.39,0.43$ \\
& pGAD & -0.02 & 0.12 & & $-0.27,0.22$ \\
Suicidal Ideation & No & - & - & - & - \\
& Yes & 0.15 & 0.17 & 0.40 & $-0.19,0.48$ \\
Age & & 0.02 & 0.00 & $<0.001$ & $0.01,0.03$ \\
Gender & Male & - & - & - & - \\
& Female & -0.08 & 0.09 & 0.38 & $-0.25,0.09$
\end{tabular}

${ }^{\star} \mathrm{p}$ value for trend. ^Not adjusted for physical condition. pMDD probable Major Depressive Disorder. pGAD probable Generalised Anxiety Disorder 
Table 4. Logistic regression model showing the relationship between depression, anxiety, suicidal ideation and wanting help with quitting, adjusting for age, gender, and physical health condition (in subset of current smokers, $\mathrm{N}=1015$ ).

\begin{tabular}{llllll}
\hline Variable & & OR & SE & $\mathbf{p}$ & $\mathbf{9 5 \% ~ C l}$ \\
\hline Depression & No symptoms & - & - & - & - \\
& Some symptoms & 1.01 & 0.22 & & $0.65,1.55$ \\
& Probable MDD & 0.94 & 0.22 & & $0.60,1.48$ \\
Anxiety^ & & & & $0.08^{*}$ & \\
& No symptoms & - & - & - & - \\
& Some symptoms & 1.40 & 0.47 & & $0.72,2.72$ \\
Suicidal Ideation & No & 1.42 & 0.30 & & $0.94,2.16$ \\
& Yosable GAD & - & - & - & - \\
Age & Yes & 1.72 & 0.50 & 0.06 & $0.98,3.03$ \\
Gender & Male & 1.00 & 0.01 & 0.91 & $0.99,1.01$ \\
& Female & - & - & - & - \\
& & 0.75 & 0.11 & 0.06 & $0.55,1.01$
\end{tabular}

${ }^{*} p$ value for linear trend. ${ }^{\wedge}$ not adjusted for physical condition. MDD probable Major Depressive Disorder. GAD probable Generalised Anxiety Disorder 